\title{
Genetic Differentiation of Puccinia triticina Populations in the Middle East and Genetic Similarity with Populations in Central Asia
}

\author{
J. A. Kolmer, M. E. Ordoñez, J. Manisterski, and Y. Anikster
}

First and second authors: United States Department of Agriculture-Agricultural Research Service Cereal Disease Laboratory, 1551 Lindig, St. Paul, MN 55108; and third and fourth authors: Institute for Cereal Crop Improvement, Tel Aviv University, Tel Aviv 69978, Israel. Accepted for publication 31 January 2011.

\section{ABSTRACT}

Kolmer, J. A., Ordoñez, M. E., Manisterski, J., and Anikster, Y. 2011. Genetic differentiation of Puccinia triticina populations in the Middle East and genetic similarity with populations in Central Asia. Phytopathology 101:870-877.

Leaf rust of wheat, caused by Puccinia triticina, is a common and widespread disease in the Middle East. The objective of this study was to determine whether genetically differentiated groups of $P$. triticina are present in the Middle East region and to compare the population from the Middle East with the previously characterized population from Central Asia to determine whether genetically similar groups of isolates are found in the two regions. In total, 118 isolates of $P$. triticina collected from common wheat and durum wheat in Egypt, Israel, Turkey, Ethiopia, and Kenya were tested for virulence on 20 lines of wheat with single genes for leaf rust resistance and for molecular genotypes with 23 simplesequence repeat (SSR) markers. After removal of isolates with identical virulence and SSR genotype in each country, 103 isolates were retained for further analysis. Clustering of SSR genotypes based on two-di- mensional principal coordinates and virulence to wheat differential lines grouped the isolates into four Middle East (ME) groups. The two largest ME groups had virulence phenotypes typical of isolates collected from common wheat and two smaller ME groups had virulence typical of isolates collected from durum wheat. All pairs of ME groups were significantly differentiated for SSR genotype based on $R_{S T}$ and $F_{S T}$ statistics, and for virulence phenotype based on $\Phi_{P T}$. All ME groups had observed values of heterozygosity greater than expected and significant fixation indices that indicated the clonal reproduction of urediniospores in the overall population. Linkage disequilibria for SSR genotypes was high across the entire population. The overall values of $R_{S T}$ and $F_{S T}$ were lower when isolates were grouped by country of origin that indicated the likely migration of isolates within the region. Although the two ME groups with virulence typical of isolates from common wheat were not differentiated for SSR genotype from groups of isolates from Central Asia based on $R_{S T}$, there was no direct evidence for migration between the two regions because all ME isolates differed from the Central Asia isolates for SSR genotypes.
Puccinia triticina Erikss., the causal agent of leaf rust of wheat, is a widespread and commonly occurring pathogen throughout the Middle East region (30,31). Leaf rust is considered a major disease of wheat in Israel (21), Egypt (23), and the coastal areas of Turkey (5). Leaf rust of wheat is most commonly found in coastal areas along the Mediterranean or Black Sea region or in river valleys where cooler temperatures and higher moisture levels are found. Common hexaploid wheat (Triticum aestivum L.) and tetraploid durum wheat (T. turgidum subsp. durum) are cultivated throughout the region. Different types of $P$. triticina that are distinct for virulence (26) and molecular genotypes (25) are specialized to common wheat and durum wheat, although the two types share the alternate host Thalictrum speciosissimum L. and are sexually compatible (2). The Fertile Crescent region of the Middle East, extending from Israel and Lebanon to the Mesopotamia region of Turkey, Syria, Iran, and Iraq, is the center of origin for modern forms of common and durum wheat (9). Arthur (4) indicated that the center of origin of P. triticina was in southwest Asia, likely somewhere in the Fertile Crescent region. T. speciosissimum is found in the Mediterranean region, including Turkey (36). Ethiopia also has a diverse wheat culture because common wheat and durum are cultivated together and land races of tetraploid emmer wheat have been grown in Ethiopia for thousands of years (28).

Corresponding author: J. A. Kolmer; E-mail address: jkolmer@umn.edu

doi:10.1094/PHYTO-10-10-0268

This article is in the public domain and not copyrightable. It may be freely reprinted with customary crediting of the source. The American Phytopathological Society, 2011
The population biology of $P$. triticina has been studied using both virulence to wheat differential lines with specific leaf rust resistance genes and various types of molecular markers. Goyeau et al. (11) found that distinct groups of P. triticina in France had a high correlation between virulence to wheat differential lines and simple-sequence repeat (SSR) markers. Similarly, a moderate correlation between virulence and SSR markers was found in $P$. triticina groups in North America (27), South America (24), and Central Asia (17). Groups of P. triticina that were collected from tetraploid durum wheat and hexaploid common wheat (22) had very high correlations between virulence phenotypes and SSR genotypes. All populations of $P$. triticina that have thus far been examined showed very strong indications of clonal reproduction, with no evidence for sexual recombination. The population genetics of clonally reproducing populations has recently been examined (12).

The major objective of this study was to determine whether distinct groups of $P$. triticina based on SSR genotypes and virulence phenotypes are present in the Middle East and East Africa. Also, because this region may include the center of origin for $P$. triticina, we examined the Middle East population for any characteristics that might indicate the presence of sexual recombination. Furthermore, we wished to determine whether the population in the Middle East where wheat has been cultivated for thousands of years had similar associations between virulence phenotype and SSR genotype that characterized populations in North America and South America, where wheat has been cultivated for a much shorter period of time and where suitable alternate hosts are not present. The final objective was to compare the $P$. triticina population from the Middle East and East Africa 
with previously characterized populations in Central Asia to assess the degree of genetic relatedness of $P$. triticina groups in these two regions.

\section{MATERIALS AND METHODS}

P. triticina isolates. Collections of $P$. triticina were obtained from Israel (34 collections), Ethiopia (19 collections), Egypt (57 collections), Turkey (8 collections), and Kenya (5 collections). Collections from common wheat in Israel and Egypt were from the mid- to late 1990s. Collections from durum wheat in Israel were from 2004. Collections from Ethiopia were from 2007, 2008, and the late 1980s. Collections from Turkey were from the late 1980s. Collections from Kenya were from the mid-1980s. A collection consisted of leaves with uredinial infections from a single cultivar or breeding line of wheat at a single location. One to two single uredinial isolates increased from each collection were derived as previously described (16). Urediniospores of most isolates had been tested previously for virulence phenotype and were kept afterward in either liquid nitrogen (23) or vacuum tubes (15) at $4{ }^{\circ} \mathrm{C}$ prior to additional virulence testing and SSR genotyping.

Virulence phenotypes. Urediniospores of each isolate were used to inoculate 7-day-old seedlings of 'Thatcher' (CI 1003) as previously described (16) in order to increase urediniospores for virulence testing and DNA extraction. To determine the virulence phenotypes of the $P$. triticina isolates, five sets of four Thatcher near-isogenic lines of wheat, each carrying one leaf rust resistance gene, were used: set 1, Lrl (isogenic line RL6003), Lr2a (RL6000), Lr2c (RL6047), and Lr3 (RL6002); set 2, Lr9 (RL6010), Lr16 (RL6005), Lr24 (RL 6064), and Lr26 (6078); set 3, Lr3ka (RL6007), Lr11 (RL6053), Lr17 (RL6008), and Lr30 (RL6049); set 4, LrB (RL6047), Lr10 (RL6004), Lrl4a (RL6013), and Lrl8 (RL6009); set 5, Lr3bg (RL6042), Lr14b (RL6006), Lr20 (RL 6092), and Lr28 (RL6079). Thatcher was included as a susceptible control. Urediniospores of each isolate were spray inoculated to each set of 7- to 8-day-old differentials as previously described (16). Virulence phenotypes were determined 10 to 12 days after inoculation for each isolate on each Thatcher differential line using a 0 -to-4 scale (20). Infection types 0 to $2^{+}$(immune response to moderate uredinia with necrosis or chlorosis) were classified as avirulent and infection types 3 to 4 (moderate to large uredinia without chlorosis or necrosis) were classified as virulent. Each isolate was given a five-letter code based on virulence or avirulence to each of the five sets of four differentials as adapted from the North American nomenclature for virulence in $P$. triticina (20). For analysis, virulence phenotypes were described with a 20-digit binary number based on avirulence or virulence.

Molecular genotypes. DNA was extracted from 25 to $30 \mathrm{mg}$ of urediniospores of each isolate by first grinding the spores with $25 \mathrm{mg}$ of glass beads in a Savant FastPrep shaker (FP120; Holbrook, NY) for $20 \mathrm{~s}$ and then using an OmniPrep extraction kit (GenoTech, St. Louis) according to instructions. In all, 1 to 2 ng of DNA was used for each polymerase chain reaction (PCR) amplification.

Twenty-three SSR microsatellite primer pairs developed from genomic libraries of $P$. triticina were used to characterize the collection: PtSSR 3, PtSSR 13, PtSSR 50, PtSSR 55, PtSSR 61, PtSSR 68-1, PtSSR 76, PtSSR 91, PtSSR 92, PtSSR 151A, PtSSR 152, PtSSR 154, PtSSR 158, PtSSR 161, PtSSR 164, PtSSR 173, PtSSR 184, PtSSR 186 (35), RB 1, RB 8, RB 11, RB 26, and RB 35 (7). Amplification and electrophoresis were carried out as previously described (35). Allele sizes in base pairs were scored visually for each primer pair by using a LI-COR (Lincoln, NE) 4200 or 4300 DNA sequencer that was calibrated with IRDye 700 molecular weight size standards. DNA bands generated by each primer pair were compared with the allele sizes in the initial characterization of the SSR primers (35) and also with other $P$. triticina isolates previously characterized (27) using the same set of SSR primers. Separate DNA samples of isolates included in both previous studies and in the current study as controls had the same SSR genotypes.

Data analysis. The molecular weights for alleles at each of the 23 SSR loci for all isolates were recorded in the GenAlex 6 (29) format. Isolates from the same country that had identical virulence phenotypes and SSR genotypes were eliminated, which left 103 isolates for further analysis. The P. triticina isolates were assigned to Middle East (ME) groups based on a principal coordinate analysis (PCA) plot derived from a matrix of genetic distances (33) between all pairs of SSR genotypes. Isolates in two of the groups were also separated based on virulence to Thatcher wheat. Neighbor-joining trees $(1,001$ total) were generated with Powermarker v3.25 (19) using Nei's distance coefficient, and bootstrap values for support of isolates in the groups were obtained with the CONSENSE program in Phylip 3.6 (10).

Averages of single-locus parameters for the isolates in the SSR groups-number of alleles, number of effective alleles $\left(N_{e}\right)$, Shannon's information index $(I)$, observed heterozygosity $\left(H_{O}\right)$, expected heterozygosity $\left(H_{E}\right)$, and fixation index $(F)$-were calculated with GenAlEx 6. Genetic differentiation via the analysis of molecular variance (AMOVA) (8) with 999 permutations of the data set was calculated for the SSR genotypes with $R_{S T}$ that assumes a stepwise mutation model and by $F_{S T}$ that assumes the infinite alleles model. An analogous measure developed for binary data, $\Phi_{P T}$, was used to calculate differentiation of the virulence phenotypes in the SSR groups. Pairwise values of $R_{S T}, F_{S T}$, and $\Phi_{P T}$ were calculated via AMOVA among SSR groups. A Mantel correlation coefficient was calculated between the SSR distance matrix and the virulence distance matrix with GenAlex 6. Linkage disequilibrium across all SSR loci was calculated with the index of association $\left(I_{A}\right)$ and also with a measure corrected for the number of loci, $\bar{r}_{D}$, using MultiLocus v1.3 software (1). Tests of departure from random mating for both indices were done with 1,000 randomizations of the data set. The significance in differences of frequency $(\%)$ of virulence to leaf rust resistance genes in different $\mathrm{SSR}$ groups of $P$. triticina isolates was determined with Fisher's exact test (34).

Ninety-nine isolates of $P$. triticina from Central Asia (Kazakhstan, Tajikistan, and Uzbekistan) and the Caspian Sea region (Azerbaijan, Armenia, and Georgia) that had been previously grouped by country of origin (17) were grouped based on a PCA plot derived from the SSR genotypes using the same set of 23 SSR markers as the isolates from the ME. Isolates from Central Asia and the Caspian Sea had also been tested for virulence to the same 20 Thatcher line differentials as the ME isolates. The data set from Central Asia and Caspian Sea region was combined with the ME data set for both SSR genotypes and virulence phenotypes. Tests of genetic differentiation of isolate groups from both regions were done in GenAlex 6 via the AMOVA procedure with 999 permutations of the combined data set. $R_{S T}$ and $F_{S T}$ were used to determine differentiation of groups based on SSR genotypes and $\Phi_{P T}$ was used to determine differentiation based on virulence phenotypes. $R_{S T}$ and $F_{S T}$ pairwise values between SSR groups from both continents were also plotted as an unrooted tree with PHYLIP 3.6 (10) using the NEIGHBOR clustering option.

\section{RESULTS}

In total, $118 P$. triticina isolates from Egypt, Israel, Ethiopia, Turkey, and Kenya were tested for SSR genotypes and virulence phenotypes. Only one isolate of each SSR genotype-virulence phenotype from each country was retained. After removal of identical clones, there were 103 isolates for the analyses. There 
were 42 isolates from Egypt, 27 from Israel, 19 from Ethiopia, 10 from Turkey, and 4 from Kenya (Table 1). The isolates were placed into ME groups using a PCA plot based on SSR genotypes, and virulence to the wheat differential lines that resulted in four groups (Fig. 1A). Isolates in ME-1 and ME-2 clustered discretely for SSR genotype and had virulence characteristics of isolates from common wheat. Isolates in ME-3 and ME-4 were well separated from isolates in ME-1 and ME-2 based on SSR genotypes and had virulence phenotypes characteristic of isolates from durum wheat. Isolates in ME-3 were avirulent to Thatcher and isolates in ME-4 were virulent to Thatcher. High bootstrap support values of $>95 \%$ were obtained for clustering of isolates within ME-3 and ME-4 and separation of ME- 1 and ME-2 from ME-3 and ME-4 (Fig. 1B). ME-1 consisted of 40 isolates from all five countries, with 39 virulence phenotypes and 18 SSR genotypes (Table 2). ME-2 was the largest group, with 47 isolates from Egypt, Israel, Kenya, and Turkey that included 41 virulence phenotypes and 23 SSR genotypes. ME-3 was the smallest group, with three isolates, all from Ethiopia, that had different SSR genotypes and were avirulent to Thatcher. ME-4 consisted of 13 isolates from Ethiopia, Egypt, Israel, and Turkey that had nine different SSR genotypes, with 10 virulence phenotypes that were mostly BBB-- phenotypes (Table 1).

The four groups of isolates varied for the average number of alleles per SSR locus from 3.30 in ME-2 to 2.21 in ME-3 (Table 3 ). The four groups varied little for number of effective alleles $\left(N_{e}\right)$, from 1.76 to 1.88 . The diversity of SSR genotypes as measured by Shannon's $I$ was somewhat higher in the two larger groups, ME-1 and ME-2, compared with the smaller groups ME-3 and ME-4. All four groups had higher values of observed heterozygosity $\left(H_{o}\right)$ at SSR loci compared with expected values $\left(H_{e}\right)$. Isolates in ME-1 and ME-2 had larger fixation indices $(F)$, which is a comparison of the observed versus expected heterozygosity levels, compared with isolates in ME-3 and ME-4. The lower ratio

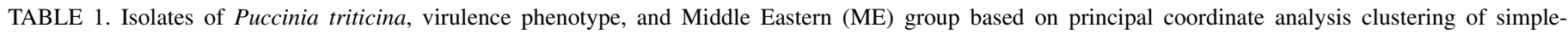
sequence repeat (SSR) genotypes

\begin{tabular}{|c|c|c|c|c|c|c|c|c|c|}
\hline Isolate no. & Country & Designation & $\begin{array}{l}\text { Virulence } \\
\text { phenotype }\end{array}$ & $\begin{array}{c}\text { ME } \\
\text { SSR group }\end{array}$ & Isolate no. & Country & Designation & $\begin{array}{l}\text { Virulence } \\
\text { phenotype }\end{array}$ & $\begin{array}{c}\text { ME } \\
\text { SSR group }\end{array}$ \\
\hline 1 & Egypt & $154-\mathrm{A}$ & MCDJT & 1 & 53 & Egypt & $31-\mathrm{A}$ & CHMNS & 2 \\
\hline 2 & Egypt & $16-\mathrm{A}$ & TDQST & 1 & 54 & Egypt & $31-C$ & THJSQ & 2 \\
\hline 3 & Egypt & $162-\mathrm{A}$ & MBDJT & 1 & 55 & Egypt & $9-A-1$ & CCPNS & 2 \\
\hline 4 & Egypt & 182-A & TCBDT & 1 & 56 & Ethiopia & $7-1-2$ & PBDSQ & 2 \\
\hline 5 & Egypt & 19-C & KBBJK & 1 & 57 & Ethiopia & $7-4-2$ & MBBSQ & 2 \\
\hline 6 & Egypt & 195-B & MFBJJ & 1 & 58 & Ethiopia & $161-\mathrm{A}$ & TCJSQ & 2 \\
\hline 7 & Egypt & $2-\mathrm{A}$ & MCJST & 1 & 59 & Ethiopia & $4026-1$ & FCFSJ & 2 \\
\hline 8 & Egypt & 202-B & MCGJK & 1 & 60 & Ethiopia & $4026-3$ & PBDSS & 2 \\
\hline 9 & Egypt & 218-A & TCPST & 1 & 61 & Ethiopia & 4164-3 & BBBGB & 2 \\
\hline 10 & Egypt & 22-B & KBBSK & 1 & 62 & Israel & $173-\mathrm{A}$ & TCDSQ & 2 \\
\hline 11 & Egypt & 223-A & MBDST & 1 & 63 & Israel & 173-B & SCPBJ & 2 \\
\hline 12 & Egypt & 24-B & LCDJT & 1 & 64 & Israel & 174-B & FBPSQ & 2 \\
\hline 13 & Egypt & $31-B$ & CCPNS & 1 & 65 & Israel & 181-B & CBDQQ & 2 \\
\hline 14 & Egypt & 31-B2 & FHMNS & 1 & 66 & Israel & 182-A & DBBQG & 2 \\
\hline 15 & Egypt & $35-A$ & MCDST & 1 & 67 & Israel & 183-A & MCBNQ & 2 \\
\hline 16 & Egypt & $35-\mathrm{B}$ & PLMLK & 1 & 68 & Israel & 4014-9 & TBMDQ & 2 \\
\hline 17 & Egypt & $37-B$ & TFBST & 1 & 69 & Israel & $4015-8$ & MBMQQ & 2 \\
\hline 18 & Egypt & $39-A$ & TDBJK & 1 & 70 & Israel & 4016-11 & CBMQQ & 2 \\
\hline 19 & Egypt & 39-B & TDBJT & 1 & 71 & Israel & D-1 & TGLQQ & 2 \\
\hline 20 & Egypt & $4-\mathrm{A}$ & PCDSR & 1 & 72 & Israel & E-1 & PGTSS & 2 \\
\hline 21 & Egypt & $53-\mathrm{B}$ & MCDDT & 1 & 73 & Israel & F-1 & PBDSQ & 2 \\
\hline 22 & Egypt & $55-\mathrm{B}$ & TCDST & 1 & 74 & Israel & $\mathrm{K}-2$ & PGBSL & 2 \\
\hline 23 & Egypt & 57-A & MFDST & 1 & 75 & Israel & L-2 & PGPSQ & 2 \\
\hline 24 & Egypt & 71-B & MDRJK & 1 & 76 & Israel & M-1 & THDSQ & 2 \\
\hline 25 & Egypt & $85-A$ & KBGJK & 1 & 77 & Israel & M-2 & PGDSQ & 2 \\
\hline 26 & Egypt & 89-B & MCDJS & 1 & 78 & Kenya & 4104-1 & FBBSS & 2 \\
\hline 27 & Egypt & $90-\mathrm{C}$ & TPMST & 1 & 79 & Kenya & 4104-3 & TBDNS & 2 \\
\hline 28 & Ethiopia & $8-2-1$ & MCDSS & 1 & 80 & Kenya & 4105-1 & CGDLB & 2 \\
\hline 29 & Ethiopia & $7-1-1$ & MCNSS & 1 & 81 & Turkey & $221-\mathrm{A}$ & PBDSQ & 2 \\
\hline 30 & Ethiopia & $8-2-1$ & MCTSS & 1 & 82 & Turkey & 222-A & FHPSQ & 2 \\
\hline 31 & Ethiopia & $7-2-3$ & MFSSS & 1 & 83 & Turkey & 222-B & CBPSQ & 2 \\
\hline 32 & Ethiopia & $7-6-1$ & TCBSK & 1 & 84 & Turkey & $222-\mathrm{C}$ & FHPNQ & 2 \\
\hline 33 & Ethiopia & $7-8-4$ & SCFLJ & 1 & 85 & Turkey & 224-A & FHPSQ & 2 \\
\hline 34 & Ethiopia & $8-3-2$ & MCFSS & 1 & 86 & Turkey & 4019-2 & FBTNQ & 2 \\
\hline 35 & Ethiopia & 4131-9 & TFDSK & 1 & 87 & Turkey & 4064-1 & FHTSQ & 2 \\
\hline 36 & Israel & 188-B & MBDJT & 1 & 88 & Ethiopia & $7-4-1$ & BBBBB & 3 \\
\hline 37 & Israel & $770-2$ & LCGKH & 1 & 89 & Ethiopia & $4090-3$ & BBBBB & 3 \\
\hline 38 & Israel & A-2 & BHBQJ & 1 & 90 & Ethiopia & 4114-7 & BBBBB & 3 \\
\hline 39 & Kenya & $84-1$ & TBDKT & 1 & 91 & Egypt & $220-B$ & BBBQJ & 4 \\
\hline 40 & Turkey & $4065-3$ & MFDJT & 1 & 92 & Ethiopia & $18-3$ & BBBQJ & 4 \\
\hline 41 & Egypt & $100-\mathrm{A}$ & TCJSQ & 2 & 93 & Ethiopia & $26-2$ & BBBGJ & 4 \\
\hline 42 & Egypt & 103-A & TCDSQ & 2 & 94 & Israel & 1492 & BBBGJ & 4 \\
\hline 43 & Egypt & $112-\mathrm{C}$ & MBDSQ & 2 & 95 & Israel & 1498 & BBBQG & 4 \\
\hline 44 & Egypt & 116-A & CGMQS & 2 & 96 & Israel & 1508 & BBBGG & 4 \\
\hline 45 & Egypt & 142-B & PCDSQ & 2 & 97 & Israel & $186 \mathrm{~A}$ & BBBQJ & 4 \\
\hline 46 & Egypt & 144-B & MGPQL & 2 & 98 & Israel & 4013-7 & DBGLJ & 4 \\
\hline 47 & Egypt & 195-A & MCDSQ & 2 & 99 & Israel & 4014-7 & LBBGJ & 4 \\
\hline 48 & Egypt & 199-B & PBDSQ & 2 & 100 & Israel & $745-1$ & BBBLG & 4 \\
\hline 49 & Egypt & 199-C & TCDSP & 2 & 101 & Israel & $\mathrm{J}-1$ & BBBBB & 4 \\
\hline 50 & Egypt & 222-A & FCPSN & 2 & 102 & Turkey & 220-B & DBBLJ & 4 \\
\hline 51 & Egypt & $3-A$ & TCDTQ & 2 & 103 & Turkey & 4066-1 & DBDLJ & 4 \\
\hline 52 & Egypt & $3-B$ & MBPSQ & 2 & & & & & \\
\hline
\end{tabular}


of $H_{o}$ to $H_{e}$ in these two groups may be a function of the smaller number of isolates. Groups ME-3 and ME-4 also had lower levels of Shannon's I, indicating an overall lower level of SSR genotype diversity relative to the number of isolates. Linkage disequilibria between SSR loci for all 103 isolates was high, with an $I_{A}$ of 6.66 and an $\bar{r}_{D}$ of 0.32 .

The overall value of $R_{S T}$ for the four ME groups was 0.632 , with $63 \%$ of the SSR variation between the groups, $16 \%$ among individual isolates within groups, and $21 \%$ within individuals. All pairs of ME groups were significantly differentiated for $R_{S T}$ at the 0.05 confidence level (Table 4 ). The overall $F_{S T}$ was 0.344 , with $25 \%$ of the variation between groups, $0 \%$ among individuals, and
$75 \%$ within individuals. All four groups were significantly differentiated for $F_{S T}$ at the 0.05 confidence level.

The four ME groups also differed for virulence to the isogenic Thatcher lines. Isolates in ME-3 were avirulent to Thatcher and, therefore, were avirulent to all of the Thatcher lines (Table 5). Isolates in ME-4 were relatively avirulent because none of the isolates had virulence to 11 of the differentials and $<10 \%$ of the isolates had virulence to four differentials. Isolates in ME-1 and ME-2 were highly polymorphic for virulence to nearly all of the resistance genes, and differed at the 0.05 confidence level for virulence to seven resistance genes and at the 0.01 confidence level for five resistance genes. ME-1 and ME-2 differed the most
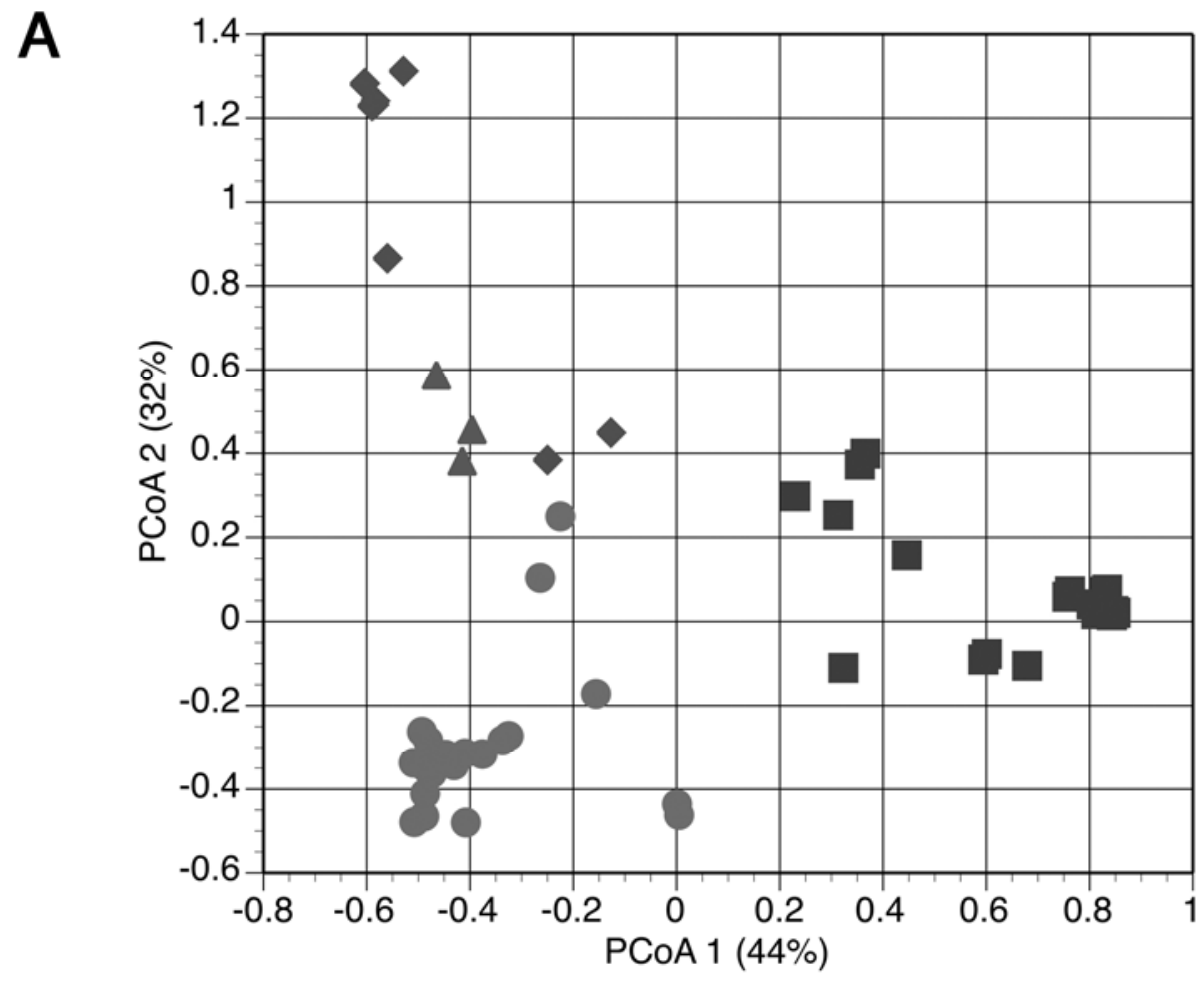

ME-1

ME-2

ME-3

ME-4

B

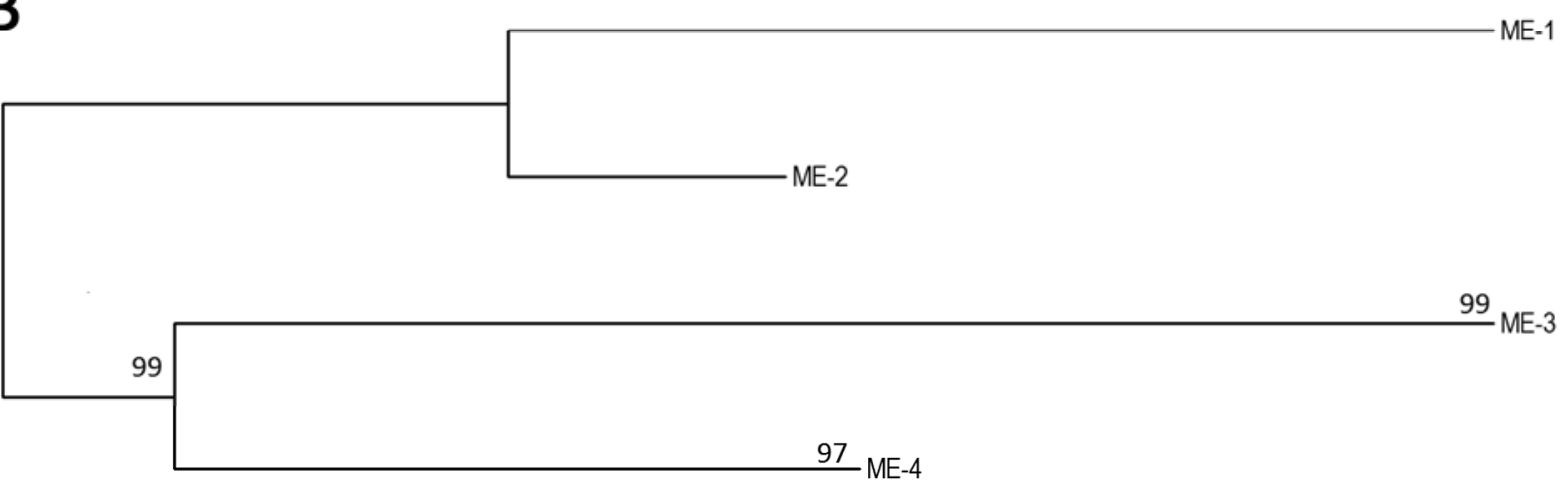

0.00

0.09

0.18

0.27

0.36

Nei's Distance

Fig. 1. A, Two-dimensional principal coordinate plot of 103 Puccinia triticina isolates from the Middle East based on simple-sequence repeat (SSR) genotypes at 23 loci. B, Neighbor-joining dendrogram of SSR genotypes in four Middle East (ME) groups based on Nei's genetic distance between groups, with bootstrap support values $>90 \%$ indicated for individual isolates within groups. 
(>70\%) for virulence to $L r 20$ and $L r 28$. The different frequencies of virulence in the ME SSR groups was also reflected in the $\Phi_{P T}$ parameters (Table 4), because all ME groups were significantly differentiated for virulence phenotypes. The overall $\Phi_{P T}$ was 0.34 , with $34 \%$ of the virulence variation between groups and $66 \%$ of the variation within groups. There was a significant correlation between the SSR genotypes and virulence phenotypes of the 103 isolates, with a Mantel value of 0.48 at the 0.001 confidence level. Removing the highly avirulent isolates in ME-3 and ME-4, the Mantel correlation of isolates in ME-1 and ME-2 for SSR genotypes and virulence phenotypes was 0.43 .

To determine whether there was a strong geographic basis for distribution of SSR genotypes and virulence phenotypes, the 103 isolates were grouped on the basis of country of origin. Isolates from Kenya and Ethiopia were grouped together. For SSR genotypes, isolates from Ethiopia and Kenya were not significantly differentiated for $R_{S T}$ from isolates from Israel and Turkey (Table 6). Isolates from Israel were not significantly differentiated from isolates from Turkey. All other pairs were significant for $R_{S T}$ differentiation. The overall $R_{S T}$ was 0.072 , with $7 \%$ of the SSR variation between countries, $27 \%$ within individual isolates, and $66 \%$ within individuals. For virulence phenotypes, isolates from Ethiopia and Kenya were not significantly differentiated for $\Phi_{P T}$ from isolates from Israel. All other pairs of countries had significant differentiation for $\Phi_{P T}$. The overall $\Phi_{P T}$ was 0.091 , with $9 \%$ of the virulence variation between countries and $91 \%$ between individual isolates.

The 99 isolates from Central Asia and the Caspian Sea region were placed into four discrete groups of SSR genotypes as represented in a PCA plot (figure not shown). Central Asia (CA) SSR groups CA-1, CA-2, CA-3, and CA-4 had 18, 46, 15, and 20 isolates, respectively. The SSR genotypes of the four ME and four CA groups were analyzed jointly to determine the degree of genetic differentiation between SSR groups in the two regions. None of the ME isolates had identical SSR genotypes with the $\mathrm{CA}$ isolates and all of the ME isolates differed from the CA isolates at four or more SSR loci. Based on $R_{S T}$ values, isolates in ME-1 were not significantly differentiated from isolates in CA-2; isolates in CA-1 and CA-4 and in CA-3 and CA-4 were not significantly differentiated; and isolates in $\mathrm{ME}-2$ were not significantly differentiated from isolates in CA-1, CA-2, and CA-3 (Fig. 2A.) All other pairs of groups were differentiated for $R_{S T}$. The overall $R_{S T}$ was 0.531 , with $0 \%$ of the variation between the two regions, $60 \%$ among $\mathrm{CA}$ and $\mathrm{ME}$ groups, $7 \%$ among individuals within groups, and 33\% within individuals (Table 7). Based on $F_{S T}$, all CA and ME groups were significantly differentiated (Fig. 2B). The overall $F_{S T}$ was 0.361 , with $8 \%$ of the variation between the two regions, $20 \%$ among the $\mathrm{CA}$ and $\mathrm{ME}$ groups, $0 \%$ among individuals, and $72 \%$ within individuals. All of the ME groups were significantly differentiated from the CA groups for virulence phenotype. The overall $\Phi_{P T}$ was 0.531 , with $1 \%$ of the virulence variation between the two regions, $39 \%$ between the CA and $\mathrm{ME}$ groups, and $60 \%$ among isolates within groups.

\section{DISCUSSION}

In this study, there was strong evidence for four distinct groups of $P$. triticina in the Middle East and East Africa, with two large groups (ME-1 and ME-2) that had SSR genotypes and virulence characteristic of isolates from common wheat cultivars and two smaller groups (ME-3 and ME-4) that were characteristic of isolates from durum wheat cultivars. These results are consistent with the strong effect of telial host specialization in structuring $P$. triticina populations in regions where durum and common wheat are grown together (22). The overall high level of linkage disequilibria for SSR loci and the $H_{o}$ values (12) of all four groups indicated high clonality in the ME population, with no evidence of sexual recombination.

The overall values for $R_{S T}$ and $F_{S T}$ were smaller when the isolates were grouped by country compared with the groupings based on SSR genotypes. This indicated that SSR genotypes that were identical or highly related could be found in more than one country. Egypt and Israel had the highest $R_{S T}$ differentiation, due to higher number of isolates from durum wheat in Israel compared with only one durum type isolate from Egypt. Turkey and

TABLE 4. $R_{S T}$ values (top diagonal) and $\Phi_{P T}$ values (bottom diagonal) of genetic differentiation of simple-sequence repeat (SSR) genotypes and virulence phenotypes of Puccinia triticina isolates, respectively, from the Middle East in groups of SSR genotypes

\begin{tabular}{lcccc}
\hline & \multicolumn{4}{c}{ Middle Eastern (ME) SSR group ${ }^{\mathrm{a}}$} \\
\cline { 2 - 5 } Group & ME-1 & ME-2 & ME-3 & ME-4 \\
\hline ME-1 & $\ldots$ & 0.336 & 0.153 & 0.833 \\
ME-2 & 0.211 & $\ldots$ & 0.246 & 0.730 \\
ME-3 & 0.540 & 0.517 & $\ldots$ & 0.634 \\
ME-4 & 0.441 & 0.442 & 0.413 & $\ldots$ \\
\hline
\end{tabular}

a All values significant at $P<0.05$.

TABLE 2. Genotypic diversity for virulence to 20 Thatcher lines of wheat with different leaf rust resistance genes and for 23 simple-sequence repeat (SSR) loci in five groups of Puccinia triticina isolates from the Middle East as grouped by SSR genotypes

\begin{tabular}{lccccr}
\hline & \multicolumn{4}{c}{ Middle Eastern (ME) group } \\
\cline { 2 - 5 } Parameters & ME-1 & ME-2 & ME-3 & ME-4 & 13 \\
Number of isolates & 40 & 47 & 3 & 10 & 103 \\
Number of virulence phenotypes & 39 & 41 & 1 & 90 \\
Number of SSR genotypes & 18 & 23 & 3 & 53 \\
\hline
\end{tabular}

TABLE 3. Average of single-locus parameters of Puccinia triticina isolates from the Middle East in groups of simple-sequence repeat (SSR) genotypes

\begin{tabular}{|c|c|c|c|c|c|}
\hline \multirow[b]{2}{*}{ Parameters $^{\mathrm{b}}$} & \multicolumn{4}{|c|}{ Middle Eastern (ME) SSR group ${ }^{\mathrm{a}}$} & \multirow[b]{2}{*}{ Total } \\
\hline & ME-1 & ME-2 & ME-3 & ME-4 & \\
\hline Number of alleles & $2.96(0.204)$ & $3.30(0.347)$ & $2.21(0.198)$ & $2.47(0.234)$ & $2.73(0.132)$ \\
\hline$N_{e}$ & $1.76(0.104)$ & $1.88(0.132)$ & $1.78(0.158)$ & $1.77(0.191)$ & $1.80(0.074)$ \\
\hline Shannon $I$ & $0.611(0.058)$ & $0.667(0.079)$ & $0.578(0.089)$ & $0.552(0.097)$ & $0.602(0.041)$ \\
\hline$H_{o}$ & $0.638(0.083)$ & $0.649(0.083)$ & $0.391(0.068)$ & $0.405(0.081)$ & $0.521(0.041)$ \\
\hline$H_{e}$ & $0.381(0.041)$ & $0.402(0.047)$ & $0.348(0.051)$ & $0.313(0.055)$ & $0.361(0.024)$ \\
\hline$F$ & $-0.507(0.083)$ & $-0.511(0.069)$ & $-0.147(0.088)$ & $-0.221(0.068)$ & $-0.364(0.041)$ \\
\hline
\end{tabular}

a Numbers in parentheses $=$ standard error.

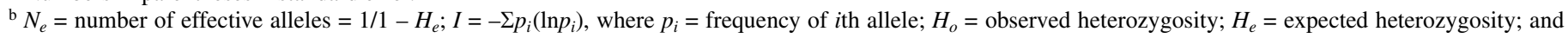
$F=$ fixation index. 
Egypt had the next highest $R_{S T}$ differentiation because isolates from Turkey were mostly in ME-2. Sampling a much larger number of collections from common wheat, McVey et al. (23) found $P$. triticina isolates with identical virulence phenotypes collected in the same year in Israel and Egypt. Collections from Egypt were more similar for virulence compared with the Israeli isolates than were isolates from Egypt and Turkey. It is very likely that some migration of $P$. triticina occurs between Israel and Egypt, and perhaps throughout the region, including East Africa.

Distinct associations between SSR genotypes and virulence phenotypes that characterized isolate groups from common wheat in North America (27) and South America (24) were not apparent in the ME population. In the North American population of $P$. triticina, isolates that were avirulent to $L r 2 a$ and virulent to $L r 2 c$ had distinct SSR genotypes compared with isolates that were virulent or avirulent to both genes. In the ME population, there was no clear distinction of SSR groups that corresponded with virulence to these genes. ME-1 and ME-2 both had isolates that were avirulent to $L r 2 a$ and virulent to $L r 2 c$ as well as isolates that were virulent or avirulent to both genes. In the ME population, isolates with virulence to $\mathrm{Lr} 17$ and $\mathrm{Lr} 3 \mathrm{bg}$ and avirulent to $\mathrm{Lr} 28$ were found in both ME-1 and ME-2 but were found in only one SSR group in North America (27) and South America (24).

Genes Lr1, Lr3, Lr10, Lr17, Lr18, Lr23, and Lr26 have been postulated to be present in Egyptian wheat cultivars (23), and Lr10, Lr11, Lr17, and Lr26 were postulated to be present in

TABLE 5. Frequencies of virulence (\%) to leaf rust resistance genes in isolates of Puccinia triticina from the Middle East in groups of simplesequence repeat (SSR) genotypes ${ }^{\mathrm{a}}$

\begin{tabular}{llllll}
\hline & \multicolumn{3}{c}{ Middle Eastern (ME) SSR groups } & \\
\cline { 2 - 4 } Gene & ME-1 & ME-2 & ME-3 & ME-4 & Difference \\
\hline Lr1 & 0.850 & 0.617 & 0.000 & 0.077 & $*$ \\
Lr2a & 0.375 & 0.255 & 0.000 & 0.000 & $\mathrm{~ns}$ \\
Lr2c & 0.450 & 0.681 & 0.000 & 0.231 & $*$ \\
Lr3 & 0.900 & 0.936 & 0.000 & 0.000 & $\mathrm{~ns}$ \\
Lr9 & 0.050 & 0.000 & 0.000 & 0.000 & $\mathrm{~ns}$ \\
Lr16 & 0.050 & 0.319 & 0.000 & 0.000 & $* *$ \\
Lr24 & 0.300 & 0.000 & 0.000 & 0.000 & $\mathrm{~ns}$ \\
Lr26 & 0.700 & 0.426 & 0.000 & 0.000 & $* *$ \\
Lr3ka & 0.250 & 0.426 & 0.000 & 0.000 & $*$ \\
Lr11 & 0.200 & 0.128 & 0.000 & 0.077 & $\mathrm{~ns}$ \\
Lr17 & 0.575 & 0.745 & 0.000 & 0.077 & $*$ \\
Lr30 & 0.225 & 0.426 & 0.000 & 0.000 & $*$ \\
LrB & 0.575 & 0.936 & 0.000 & 0.538 & $*$ \\
Lr10 & 0.850 & 0.809 & 0.000 & 0.615 & $\mathrm{~ns}$ \\
Lr14a & 0.925 & 0.787 & 0.000 & 0.077 & $\mathrm{~ns}$ \\
Lr18 & 0.050 & 0.021 & 0.000 & 0.000 & $\mathrm{~ns}$ \\
Lr3bg & 0.675 & 0.894 & 0.000 & 0.000 & $* *$ \\
Lr14b & 1.000 & 0.872 & 0.000 & 0.923 & $*$ \\
Lr20 & 0.950 & 0.234 & 0.000 & 0.692 & $* *$ \\
Lr28 & 0.725 & 0.021 & 0.000 & 0.000 & $* *$ \\
\hline
\end{tabular}

a Number of isolates in each group: ME-1 $=40, \mathrm{ME}-2=47, \mathrm{ME}-3=3$, and ME- $4=13$.

${ }^{\mathrm{b}}$ Significance of difference. ME-1 and ME-2 difference: $\mathrm{ns}=$ frequencies of ME-1 and ME-2 not significantly different, $P>0.05$; * = significant difference at $P<0.05$; ** $=$ significant difference at $P<0.01$ from Fisher's exact test (34).
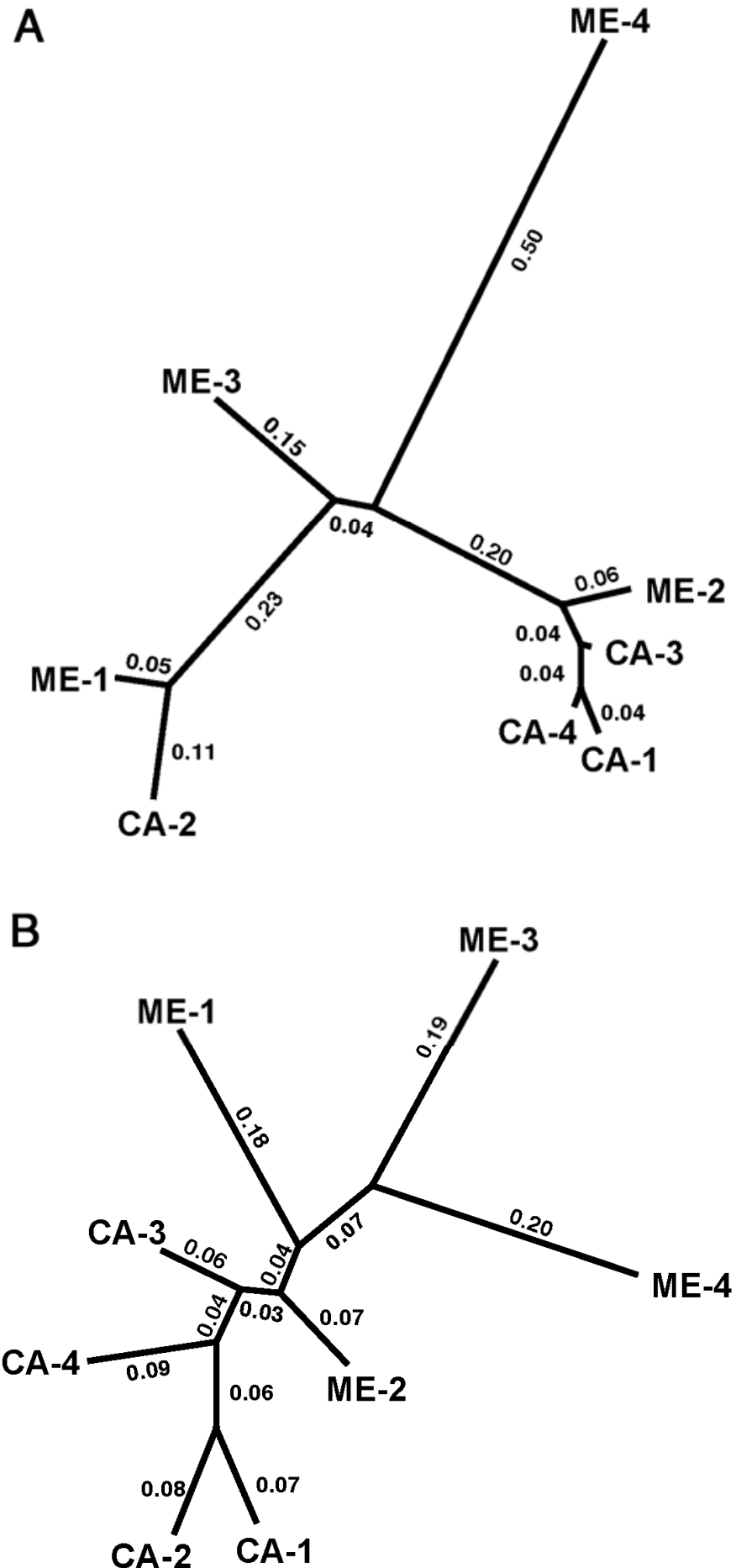

Fig. 2. Neighbor-joining plots of genetic differentiation between groups of Puccinia triticina isolates from the Middle East (ME) and Central Asia (CA). Values of genetic differentiation were derived with 999 permutations of the data set in GeneAlex 6 (29). A, Distance based on $R_{S T} ; \mathbf{B}$, distance based on $F_{S T}$.

TABLE 6. $R_{S T}$ values (top diagonal) and $\Phi_{P T}$ values (bottom diagonal) of genetic differentiation of simple-sequence repeat (SSR) genotypes and virulence phenotypes of Puccinia triticina isolates, respectively from the Middle East and East Africa

\begin{tabular}{llcccc}
\hline \multirow{2}{*}{$\begin{array}{l}\text { Number of } \\
\text { isolates }\end{array}$} & Countries & Egypt & Ethiopia-Kenya & Israel & \multicolumn{2}{c}{ Countries $^{\mathrm{a}}$} \\
\cline { 2 - 6 } & Egypt & $\ldots$ & $0.037^{*}$ & $0.172^{*}$ & 0.011 \\
23 & Ethiopia-Kenya & $0.049^{*}$ & $\ldots$ & $\ldots$ & $0.006^{*}$ \\
27 & Israel & $0.132^{*}$ & 0.020 & $\ldots .016$ \\
10 & Turkey & $0.145^{*}$ & $0.112^{*}$ & $0.099^{*}$ & $\ldots$ \\
\hline
\end{tabular}

a Asterisk $(*)=$ significant difference at $P<0.05$. 
Israeli wheats (J. A. Kolmer, unpublished data). Virulence was common in both ME-1 and ME-2 to all of these genes, except for $L r 23$, which was not included in this study, and virulence to $\operatorname{Lr} 18$, which was low in both groups. None of the isolates in ME-2 were virulent to $\operatorname{Lr} 9$ or $\operatorname{Lr} 24$. Both of these genes were introgressed into common wheat from lower ploidy relatives of wheat that are not hosts of $P$. triticina (2). In the absence of wheat cultivars with these genes, isolates with virulence to $\operatorname{Lr} 9$ and $L r 24$ would be rare. Similarly, $\operatorname{Lr} 18$ was also derived from a wild relative of wheat.

The overall genetic differentiation based on $R_{S T}$ differed compared with $F_{S T}$ for both the ME population and the combined MECA populations. $R_{S T}$, based on the single-step mutation model (32), assumes that alleles that are closer for molecular weight are more closely related. $F_{S T}$, based on the infinite-alleles model (38), assumes that all alleles, regardless of molecular weight, are equally different. Mutation is the presumed source of variation in the $R_{S T}$ model and genetic drift is the primary source in the $F_{S T}$ model. Mutation is likely a recurrent event in $P$. triticina populations because new virulence phenotypes are often detected shortly after release of wheat cultivars with race-specific $L r$ genes (14). Given the size of $P$. triticina populations (hundreds to thousands of infections per plant; hundreds to thousands of spores produced per infection, and widespread geographical distribution), it would be expected that random mutations would produce new virulence phenotypes and also variation at microsatellite loci. Single virulence differences and single SSR allele differences were found among isolates within North American groups of P. triticina (27), suggesting the importance of mutation. Genetic drift might be important in structuring $P$. triticina populations if some bottleneck effect occurred such as severe winters that would eliminate a population from overwintering, as occurred with $P$. striiformis (stripe rust of wheat) in Denmark (13), or large-scale release of cultivars that are highly resistant to all current races and removal of the alternate host, as has occurred with $P$. graminis (wheat stem rust) in North America (14). However, there is no evidence that events similar to these have influenced genetic diversity in $P$. triticina in the ME or other regions where the rust occurs.

Based on $R_{S T}$, isolates in ME-1 and ME-2 were not differentiated from groups in the CA population; however, none of the isolates in ME-1 or ME-2 had identical SSR genotypes with the $\mathrm{CA}$ isolates and, therefore, there is no direct evidence of migration between the two regions. If migration events have occurred, this was likely followed by mutation at SSR and virulence loci to generate the dissimilar genotypes. It is also possible that the major groups of $P$. triticina on common wheat in the Middle East and Central Asia were established from very similar founding populations with little current migration. The $F_{S T}$ data did not support migration between the two regions because all the ME groups were differentiated from the CA groups. There is no previous data regarding movement of $P$. triticina between the ME and CA regions.

The Middle East is unique because progenitors of cultivated wheat are found throughout the region. Triticum turgidum var. dicoccoides Koern (wild emmer), the tetraploid ( $\mathrm{AB}$ genomes) progenitor of modern durum and common wheat, is native to the region and grows in Israel, Jordan, Syria, and Turkey. Archeological sites have indicated that wild emmer has been cultivated for over 10,000 years in the Fertile Crescent (9). Isolates of $P$. triticina from common wheat are virulent to most accessions of $T$. turgidum var. dicoccoides (3); therefore, it is possible that leaf rust has been established in the Middle East for thousands of years. The majority of isolates of $P$. triticina collected from stands of wild emmer in Israel had virulence phenotypes (6) similar to isolates in ME-1 and ME-2. The P. triticina collections from wild emmer did not differ for virulence phenotype compared with isolates from common wheat $(3,18)$ in Israel. Infections of $P$. triticina potentially survive the summer on $T$. dicoccoides, providing an inoculum source for infections on cultivated wheat in the fall and spring.

The long-term cultivation of emmer wheat combined with the use of modern common wheat and durum wheat cultivars has the potential to host a highly diverse population of $P$. triticina in the ME region. The ME population was unique compared with other $P$. triticina populations because characteristic associations between certain virulence phenotypes and SSR groups were not found. The long-term establishment of $P$. triticina in this region may have reduced disequilibria between these characteristic virulence and SSR markers. It is also possible that the widespread cultivation of modern hexaploid common wheat cultivars throughout the ME region has had a major selective effect and has reduced whatever effect the wild emmer populations had on the $P$. triticina population.

Alternate hosts for $P$. triticina are not native to Israel (37), Egypt, Ethiopia, or Kenya. Thalictrum flavum (syn. T. speciosissimum) has been reported in Turkey (36); however, the pycniaaecial stage of $P$. triticina has not been observed and there is no evidence that $T$. flavum is functioning as an alternate host. Wild emmer and T. urartu, the donor of the A genome in common wheat and durum wheat, and T. tauschii, the donor of the D genome, are all found in Turkey (9). The overlapping range of wheat progenitors with a suitable alternate host indicates that Turkey may be the center of origin for $P$. triticina. If sexual recombination occurs in Turkey on $T$. flavum, the effects on population parameters are very small compared with the clonal effect of urediniospore production and selection by leaf rust resistance genes in the wheat crop. Furthermore, there was no evidence to indicate the importance of sexual recombination because high $H_{o}$ and $F$ values in ME-1 and ME-2 indicated clonal reproduction.

TABLE 7. Analysis of variance for simple-sequence repeat (SSR) allele variation and virulence variation in isolates of Puccinia triticina from the Middle East and Central Asia

\begin{tabular}{|c|c|c|c|c|}
\hline Source of variance & Variance & Total variance $(\%)$ & Differentiation statistic & Probability \\
\hline \multicolumn{5}{|l|}{ SSR allele variation } \\
\hline Among regions & 0 & 0 & $R_{R T} 0.000$ & 1.000 \\
\hline Among groups & 11,067 & 60 & $R_{S T} 0.599$ & 0.001 \\
\hline Within individuals & 6,085 & 33 & $R_{I T} 0.178$ & 0.001 \\
\hline \multicolumn{5}{|l|}{ Virulence variation } \\
\hline Among regions & 0.71 & 8 & $F_{S R} 0.286$ & 0.001 \\
\hline Among groups & 1.75 & 20 & $F_{S T} 0.361$ & 0.001 \\
\hline \multicolumn{5}{|l|}{ Virulence variation } \\
\hline Among regions & 0.03 & 1 & $\Phi_{R T} 0.009$ & 0.042 \\
\hline Among groups & 1.45 & 39 & $\Phi_{P R} 0.393$ & 0.001 \\
\hline Within groups & 2.20 & 60 & $\Phi_{P T} 0.398$ & 0.001 \\
\hline
\end{tabular}


In summary, two groups that had virulence phenotypes and SSR genotypes characteristic of isolates from common wheat and two groups typical of isolates from durum wheat characterized the $P$. triticina population in the Middle East. Although virulenceSSR genotype associations characteristic of $P$. triticina populations in other regions were not found and an alternate host of $P$. triticina is present in the region, suggesting the possibility of sexual recombination, the overall high levels of linkage disequilibria and heterozygosity at SSR loci indicated the prevalence of clonal reproduction in the ME population. Furthermore, based on $R_{S T}$ differentiation, the two groups from common wheat hosts were closely related to groups of isolates from Central Asia, although there was no direct evidence for migration due to the lack of common SSR genotypes in the two regions.

\section{LITERATURE CITED}

1. Agapow, P. M., and Burt, A. 2001. Indices of multilocus linkage disequilibrium. Mol. Ecol. Notes 1:101-102.

2. Anikster, Y., Bushnell, W. R., Eilam, T., Manisterski, J., and Roelfs, A. P. 1997. Puccinia recondita causing leaf rust on cultivated wheats, wild wheats, and rye. Can. J. Bot. 75:2082-2095.

3. Anikster, Y., Manisterski, J., Long, D. L., and Leonard, K. J. 2005. Resistance to leaf rust, stripe rust, and stem rust in Aegilops spp. in Israel. Plant Dis. 89:303-308.

4. Arthur, J. C. 1929. The Plant Rusts (Urediniales). John Wiley \& Sons, New York.

5. Braun, H. J., Zencirci, N., Altay, F., Atli, A., Avci, M., Eser, V., Kambertay, M., and Payne, T. S. 2001. Turkish wheat pool. Pages 851879 in: The World Wheat Book: A History of Wheat Breeding. A. Bonjean and W. Angus, eds. Lavoisier, Paris.

6. Dinoor, A., Eshed, N., Ecker, R., Grerechter-Amitai, Z., Solel, Z., Manisterski, J., and Anikster, Y. 1991. Fungal diseases of wild tetraploid wheat in a natural stand in northern Israel. Israel J. Bot. 40:481-500.

7. Duan, X., Enjalbert, J., Vautrin, D., Solignac, C., and Giraud, T. 2003. Isolation of 12 microsatellite loci, using an enrichment protocol, in the phytopathogenic fungus Puccinia triticina. Mol. Ecol. Notes 3:65-67.

8. Excoffier, L., Smouse, P. E., and Quattro, J. M. 1992. Analysis of molecular variance inferred from metric distances among DNA haplotypes: Application to human mitochondrial DNA restriction data. Genetics 131:479-491.

9. Feldman, M. W. 2001. Origin of cultivated wheat. Pages 3-58 in: The World Wheat Book: A History of Wheat Breeding. A. Bonjean and W. Angus, eds. Lavoisier, Paris.

10. Felsenstein, J. 1989. PHYLIP Phylogeny inference package (version 3.2). Cladistics 5:164-166.

11. Goyeau, H., Halkett, F., Zapater, M. F., Carlier, J., and Lannou, C. 2007. Clonality and host selection in the wheat pathogenic fungus Puccinia triticina. Fungal Genet. Biol. 44:474-483.

12. Halkett, F., Simon, J. C., and Balloux, F. 2005. Tackling the population genetics of clonal and partially clonal organisms. Trends Ecol. Evol. 20:194-201.

13. Justesen, A. F., Ridout, C. J., and Hovmoller, M. S. 2002. The recent history of Puccinia striiformis f. sp. tritici in Denmark as revealed by disease incidence and AFLP markers. Plant Pathol. 51:13-23.

14. Kolmer, J., Chen, X., and Jin, Y. 2009. Diseases which challenge global wheat production - the wheat rusts. Pages 89-124 in: Wheat: Science and Trade. B. F. Carver, ed. Wiley-Blackwell, Ames, IA.

15. Kolmer, J. A., and Liu, J. Q. 2000. Virulence and molecular polymorphism in international collections of the wheat leaf rust fungus Puccinia triticina. Phytopathology 90:427-436.

16. Kolmer, J. A., Long, D. L., and Hughes, M. E. 2009. Physiologic specialization of Puccinia triticina on wheat in the United States in 2007. Plant Dis. 93:538-544

17. Kolmer, J. A., and Ordoñez, M. E. 2007. Genetic differentiation of Puccinia triticina populations in Central Asia and the Caucasus. Phytopathology 97:1141-1149.

18. Kosman, E., Pardes, E., Anikster, Y., Manisterski, J., Ben Yehuda, P., Szabo, L. S., and Sharon, A. 2004. Genetic variation and virulence on Lr26 in Puccinia triticina. Phytopathology 94:632-640.

19. Liu, K., and Muse, S. V. 2005. PowerMarker: Integrated analysis environment for genetic marker data. Bioinformatics 21:2128-2129.

20. Long, D. L., and Kolmer, J. A. 1989. A North American system of nomenclature for Puccinia recondita f. sp. tritici. Phytopathology 79:525529.

21. Manisterski, J., Eyal, Z., Ben-Yehuda, P., and Kosman, E. 2000. Comparative analysis of indices in the study of virulence diversity between and within populations of Puccinia recondita f. sp. tritici in Israel. Phytopathology 90:601-607.

22. Mantovani, P., Maccaferri, M., Tubersoa, R., and Kolmer, J. 2010. Virulence phenotypes and molecular genotypes in collections of Puccinia triticina from Italy. Plant Dis. 94:420-424.

23. McVey, D. V., Nazim, M., Leonard, K. J., and Long, D. L. 2004. Patterns of virulence diversity in Puccinia triticina on wheat in Egypt and the United States in 1998-2000. Plant Dis. 88:271-279.

24. Ordoñez, M. E., German, S. E., and Kolmer, J. A. 2010. Genetic differentiation within the Puccinia triticina population in South America and comparison with the North American population suggests common ancestry and intercontinental migration. Phytopathology 100:376-383.

25. Ordoñez, M. E., and Kolmer, J. A. 2007. Simple sequence repeat diversity of a world-wide collection of Puccinia triticina from durum wheat. Phytopathology 97:574-583.

26. Ordoñez, M. E., and Kolmer, J. A. 2007. Virulence phenotypes of a worldwide collection of Puccinia triticina from durum wheat. Phytopathology 97:344-351.

27. Ordoñez, M. E., and Kolmer, J. A. 2009. Differentiation of molecular genotypes and virulence phenotypes of Puccinia triticina from common wheat in North America. Phytopathology 99:750-758.

28. Payne, T. S., Wanjema, J. K., and Girma, B. 2001. Eastern Africa wheat pool. Pages 901-938 in: The World Wheat Book: A History of Wheat Breeding. A. Bonjean and W. Angus, eds. Lavoisier, Paris.

29. Peakall, R., and Smouse, P. E. 2006. GENALEX 6: Genetic analysis in Excel. Population genetic software for teaching and research. Mol. Ecol. Notes 6:288-295.

30. Roelfs, A. P., Singh, R. P., and Saari, E. E. 1992. Rust Diseases of Wheat: Concepts and Methods of Disease Management. CIMMYT, Mexico, D.F.

31. Saari, E. E., and Prescott, J. M. 1985. World distribution in relation to economic losses. Pages 259-298 in: The Cereal Rusts. A. P. Roelfs and W. R. Bushnell, eds. Academic Press, Orlando, FL.

32. Slatkin, M. 1995. A measure of population subdivision based on microsatellite allele frequencies. Genetics 159:457-462.

33. Smouse, P. E., and Peakall, R. 1999. Spatial autocorrelation analysis of individual multiallele and multilocus genetic structure. Heredity 82:561573.

34. Steel, R. D., and Torrie, J. H. 1980. Principles and Procedures of Statistics, 2nd ed. McGraw-Hill, New York.

35. Szabo, L. S., and Kolmer, J. A. 2007. Development of simple sequence repeat markers for the plant pathogenic rust fungus Puccinia triticina. Mol. Ecol. Notes 7:708-710.

36. Tatlidil, S., Bicakci, A., Malyer, H., and Can Baser, K. H. 2005. Pollen morphology of Thalictrum L., species (Ranunculaceae) in Turkey. Pak. J. Bot. 37:203-212

37. Wahl, I., Anikster, Y., Manisterski, J., and Segal, A. 1984. Evolution at the center of origin. Pages 39-72 in: The Cereal Rusts, Vol. I. W. R. Bushnell and A. P. Roelfs, eds. Academic Press, Orlando, FL.

38. Wright, S. 1951. The genetical structure of populations. Ann. Eugen. 15:323-354. 